\title{
Selective Detection of Nitroexplosives Using Molecular Recognition within Self-Assembled Plasmonic Nanojunctions
}

\author{
Weng-I Katherine Chio, ${ }^{\dagger \dagger}$ William J. Peveler, ${ }^{\S \odot}$ Khaleel I. Assaf, $^{\perp}{ }^{\circledR}$ Suresh Moorthy,

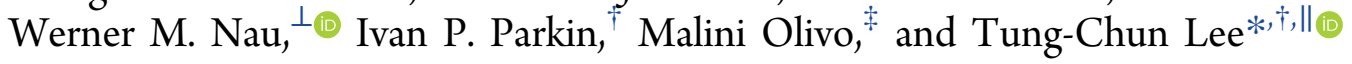 \\ ${ }^{\dagger}$ Department of Chemistry, University College London (UCL), London WC1H 0AJ, U.K. \\ ${ }^{\ddagger}$ Singapore Bioimaging Consortium (SBIC), Agency for Science Technology and Research (A*STAR), Singapore 138667, \\ Singapore \\ ${ }^{\S}$ School of Chemistry, Joseph Black Building, University of Glasgow, Glasgow G12 8QQ, U.K. \\ ${ }^{\perp}$ Department of Life Sciences and Chemistry, Jacobs University Bremen, Campus Ring 1, D-28759 Bremen, Germany \\ "Institute for Materials Discovery, University College London (UCL), London WC1H OAJ, U.K.
}

\section{Supporting Information}

ABSTRACT: We demonstrate that the reproducibility of sensors for nitroaromatics based on surface-enhanced Raman spectroscopy (SERS) can be significantly improved via a hierarchical aqueous self-assembly approach mediated by the multifunctional macrocyclic molecule cucurbit[7] uril ( $\mathrm{CB}[7])$. Our approach is enabled by the novel host-guest complexation between $\mathrm{CB}[7]$ and an explosive marker 2,4-dinitrotoluene

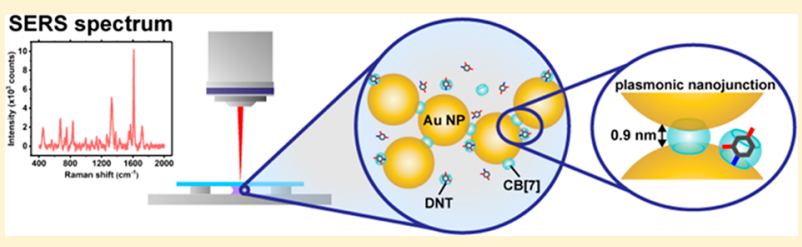
(DNT). Binding studies are performed using experimental and computation techniques to quantify key binding parameters for the first time. This supramolecular complexation allows DNT to be positioned in close proximity to the plasmonic hotspots within aggregates of $\mathrm{CB}[7]$ and gold nanoparticles, resulting in significant SERS signals with a detection limit of $\sim 1 \mu \mathrm{M}$. The supramolecular ensemble is selective against a structurally similar nitroaromatics owing to the molecular-recognition nature of the complexation as well as tolerant against the presence of model organic contaminants that bind strongly to the SERS substrates.

\section{INTRODUCTION}

Detection of trace explosives is a major challenge in both homeland security and environmental monitoring of water quality. ${ }^{1-9}$ Nitroaromatic compounds including 2,4-dinitrotoluene (DNT) and 2,4,6-trinitrotoluene (TNT), which are associated with landmines and bombs used in military activities and terrorist attacks, ${ }^{6-9}$ are present as contaminants in groundwater and soils at munitions processing sites and military ranges. ${ }^{10,11} \mathrm{DNT}$ is also a major degradation product of TNT with much higher vapor pressure. ${ }^{12}$ A highly sensitive and selective sensor is of great importance due to increasing global threats of terrorism and environmental safety concerns. Common explosive sensors have utilized mass spectrometric, fluorescent, colorimetric, electrochemical, or antibody-based techniques, ${ }^{13-16}$ but not all methods allow explosive detection on site in real time with high performance.

Surface-enhanced Raman spectroscopy (SERS) is a highly sensitive and selective spectroscopic technique used to identify analyte molecules via their vibrational fingerprints. This technique takes advantage of the strong field enhancement of plasmonic nanostructures (aka. SERS substrates) that can enhance Raman signals of molecules in their proximity by 4 to 10 orders of magnitude. ${ }^{17}$ SERS substrates based on noble metal nanoparticles such as gold (Au NPs) and silver (Ag NPs) offer numerous advantages for explosive detection such as high chemical stability, cost-effectiveness, rapid response, and little or no sample preparation. ${ }^{5,18,19}$ Attachment of macrocyclic molecules onto Au NPs can enhance sensitivity by controlling interparticle spacing and localizing analyte molecules at the plasmonic hotspots via formation of hostguest complexes. ${ }^{20-22}$

Cucurbit $[n]$ urils $(\mathrm{CB}[n]$ s where $n=5-8,10)$ are macrocyclic, rigid, highly symmetric, and Raman-active molecules, which can trap small analyte molecules inside their hydrophobic cavity to form inclusion complexes. ${ }^{23} \mathrm{CBs}$ can also bind to the surface of $\mathrm{Au}$ NPs through their electron-rich carbonyl portals, mediating the formation of precise plasmonic hotspots via aqueous self-assembly. ${ }^{20,22}$ In this context, Mahajan and Scherman reported ${ }^{24}$ the first Raman and SERS study to differentiate major $\mathrm{CB}[n]$ homologues using commercial SERS substrates. In a follow-up work, Mahajan et al. investigated ${ }^{22}$ the kinetics of $\mathrm{CB}[5]-\mathrm{Au} \mathrm{NP}$ aggregation and demonstrated the specificity of host-guest binding of $\mathrm{CB}[n]$ via solutionphase SERS measurements. Subsequent SERS studies based on $\mathrm{CB}[n]-\mathrm{Au} \mathrm{NP}$ aggregates include ferrocene, ${ }^{25}$ polycyclic aromatics, $^{26}$ diaminostilbene, ${ }^{27}$ and neurotransmitters. ${ }^{28}$

Received: March 13, 2019

Revised: April 23, 2019

Published: April 23, 2019 
(a)

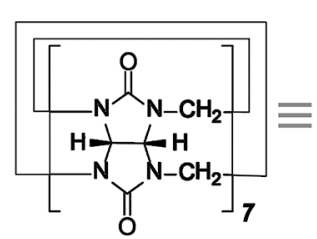

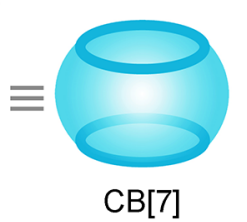

$\mathrm{CB}[7]$

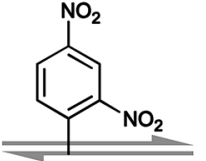

DNT
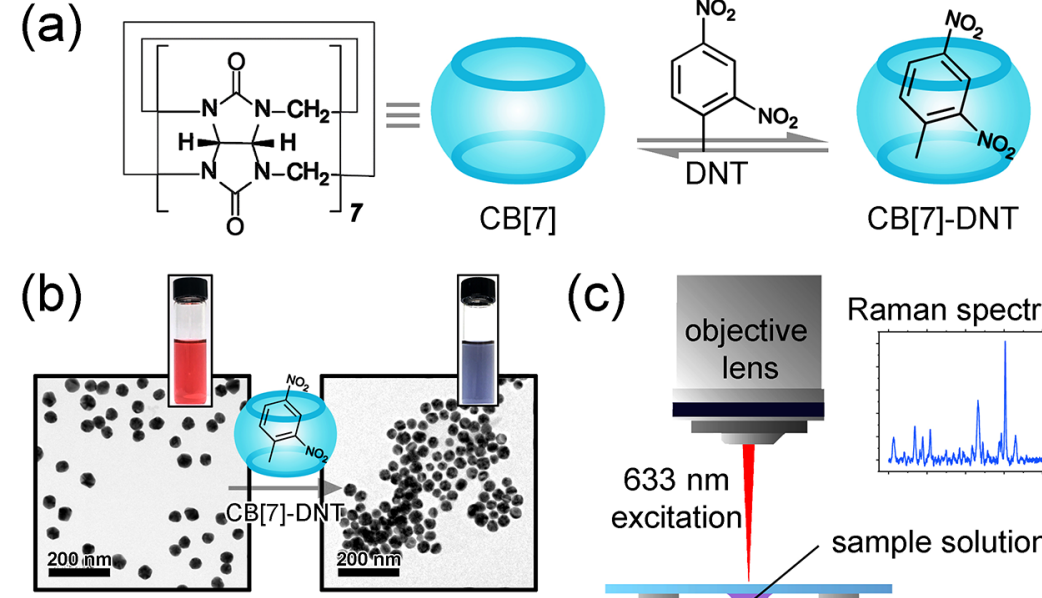

(c)
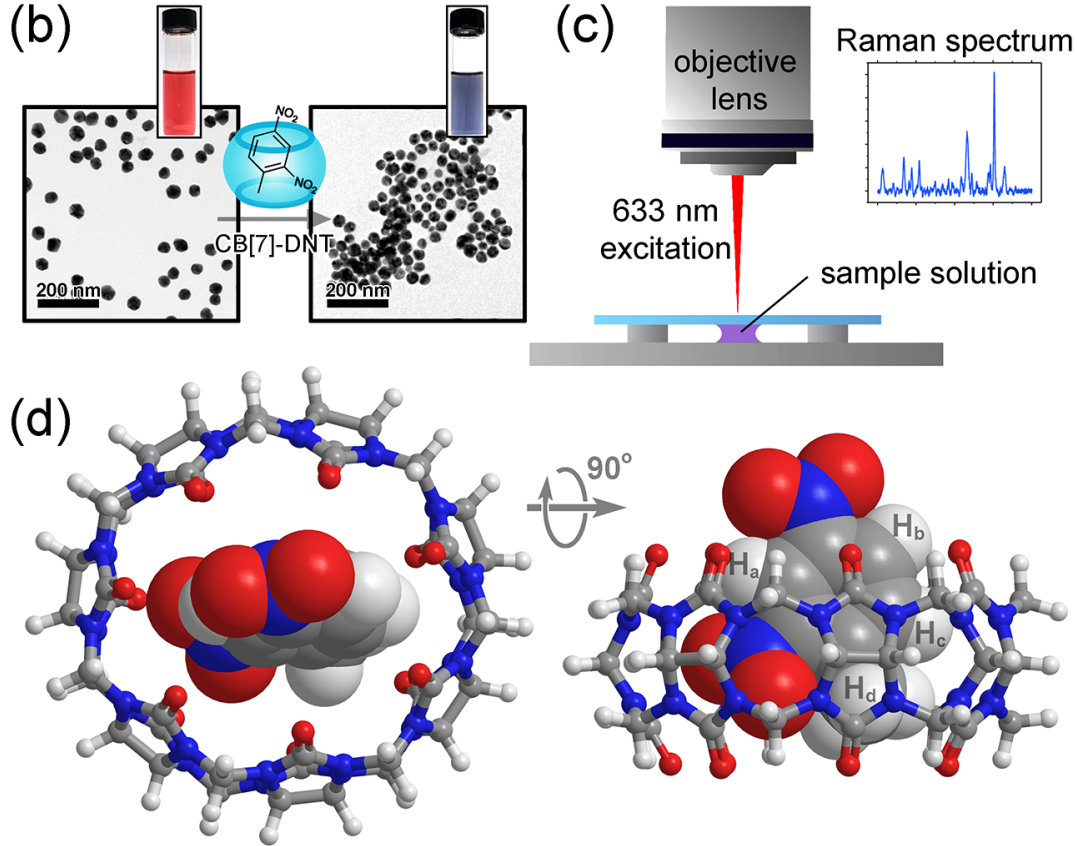

Figure 1. (a) Formation of a 1:1 inclusion complex between DNT and CB[7]. (b) TEM images and photographs showing $40 \mathrm{~nm}$ Au NPs before and after addition of the CB[7]-DNT complexes (see Figure S1 for a zoom-in). (c) Schematics of the Raman measurement; a droplet of sample solution is loaded in a custom-made cell and observed under a Raman microscope with $633 \mathrm{~nm}$ laser excitation. (d) Top and side views of an energy-minimized $\mathrm{CB}[7]-\mathrm{DNT}$ complex in water modeled at the $\mathrm{CPCM} / \mathrm{wB} 97 \mathrm{XD} / 6-311+\mathrm{G}^{* *}$ level of theory.

These previous studies focus on good CB guests that are positively charged and fit entirely or mostly within the $\mathrm{CB}$ cavity. Weaker guests, in particular polysubstituted nitroaromatics that are neutral and bulky, remain largely unexplored in such sensing systems, despite being attractive analytical targets. ${ }^{7}$

Herein we investigated the host-guest complexation of an explosive marker DNT and $\mathrm{CB}[7]$ in water using experimental and computational techniques to quantify key binding parameters of the resultant inclusion complex for the first time (Figure 1). We demonstrate the detection of DNT at different concentrations (down to $1 \mu \mathrm{M}$ ) with highly reproducible SERS signals via host-guest complexation with $\mathrm{CB}[7]$ on $\mathrm{Au}$ NPs. In addition, the new sensor exhibits high selectivity against a similar explosive molecule, picric acid, which is too bulky to bind into the $\mathrm{CB}[7]$ cavity. Notably, despite being a well-established property of CBs, molecular recognition phenomena in $\mathrm{CB}-\mathrm{Au} \mathrm{NP}$ systems have not been systematically investigated using SERS since the first experimental observation. ${ }^{22}$ Finally, the sensor also shows good tolerance against model organic contaminants that bind strongly to Au NPs.

\section{MATERIALS AND METHODS}

Materials. Citrate-stabilized gold nanoparticles (Au NPs, $40 \mathrm{~nm}$ ) were purchased from nanoComposix. $\mathrm{HCl}$, paraformaldehyde, 2,4-dinitrotoluene (DNT), 1-adamantylamine $\left(\mathrm{AdNH}_{2}\right)$, picric acid (PA, safety note: PA should be kept wetted at all times), 4-mercaptophenylboronic acid (4-MPBA), and 4-mercaptobenzonic acid (4-MBA) were purchased from Sigma-Aldrich. Methanol and ethanol were purchased from VWR. Glycoluril was purchased from Acros Organics. $\mathrm{NaCl}$ was purchased from Kanto Chemical. Cucurbit[7] uril (CB[7]) was synthesized according to the literature. ${ }^{29}$ All chemicals were used as received without further purification. Milli-Q water was used in all experiments.

Transmission Electron Microscopy (TEM). A $100 \mu \mathrm{M}$ $\mathrm{CB}[7]-\mathrm{DNT}$ stock solution was diluted 10-fold into a Au NP solution to form Au NP-CB[7]-DNT aggregates. Then, one or two $5 \mu \mathrm{L}$ drops of the solution were drop-casted onto a $\mathrm{C}$ coated 300-mesh $\mathrm{Cu}$ grid and dried with Ar gas. The images were taken using a JEOL JEM-2100 TEM at an accelerating voltage of $200 \mathrm{kV}$.

Nuclear Magnetic Resonance (NMR) Spectroscopy. $\mathrm{CB}$ [7] $(2 \mathrm{mM})$ solutions were prepared in $\mathrm{D}_{2} \mathrm{O}$. DNT was added to the solutions with 1:1 or 1.2:1 molar ratios. Similarly, PA was added to the solution with 1:1 molar ratio. The samples were sonicated for $3 \mathrm{~h} .{ }^{1} \mathrm{H}$ NMR spectra were measured on a Bruker Advance III 600 Cryo spectrometer. Chemical shifts (in ppm) were referenced to $\mathrm{D}_{2} \mathrm{O}$ with $\delta=$ 4.79 ppm for ${ }^{1} \mathrm{H}$.

Simulations. DFT calculations were performed using a Spartan'16 Parallel Suite, and force-field calculations were performed using Chem3D. Geometry optimization was performed first using MMFF94, followed by full optimization at the required level of theory $\left(\mathrm{HF} / 3-21 \mathrm{G}, \mathrm{wB} 97 \mathrm{X}-\mathrm{D} / 6-31 \mathrm{G}^{*}\right.$, 

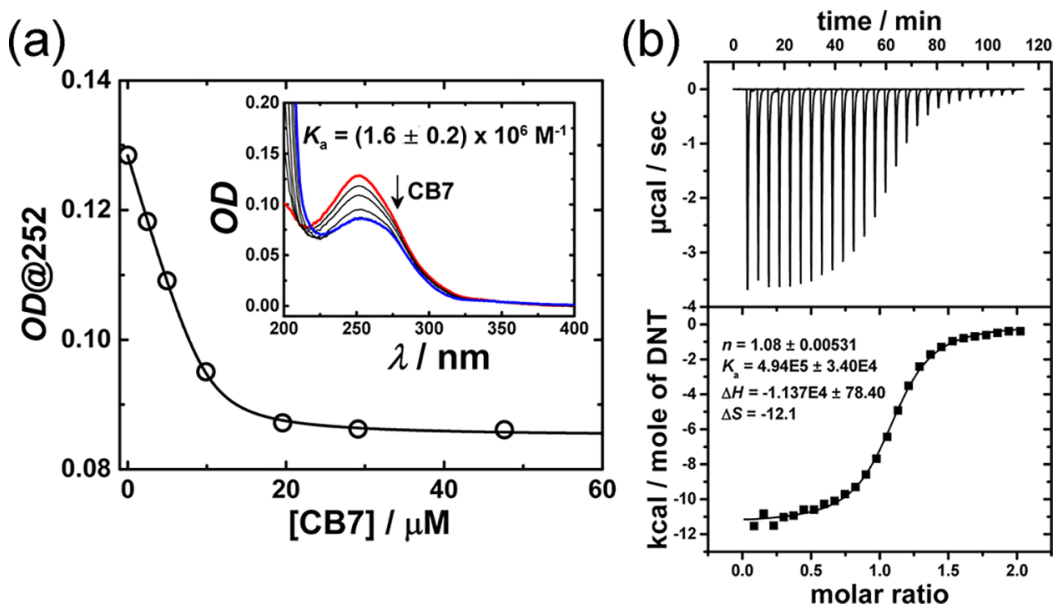

Figure 2. (a) UV-vis titration of $10 \mu \mathrm{M}$ DNT with $\mathrm{CB}$ [7]; the fitting was done by assuming a 1:1 binding model from which the binding constant was derived. Inset: Actual changes in the UV-vis spectrum with increasing concentration of $\mathrm{CB}[7]$. (b) Microcalorimetric titration results in neat water: Raw ITC data for sequential injections of $1.0 \mathrm{mM} \mathrm{CB}$ [7] into a $0.1 \mathrm{mM} \mathrm{DNT}$ solution and apparent reaction heats obtained from the integration of the calorimetric traces.
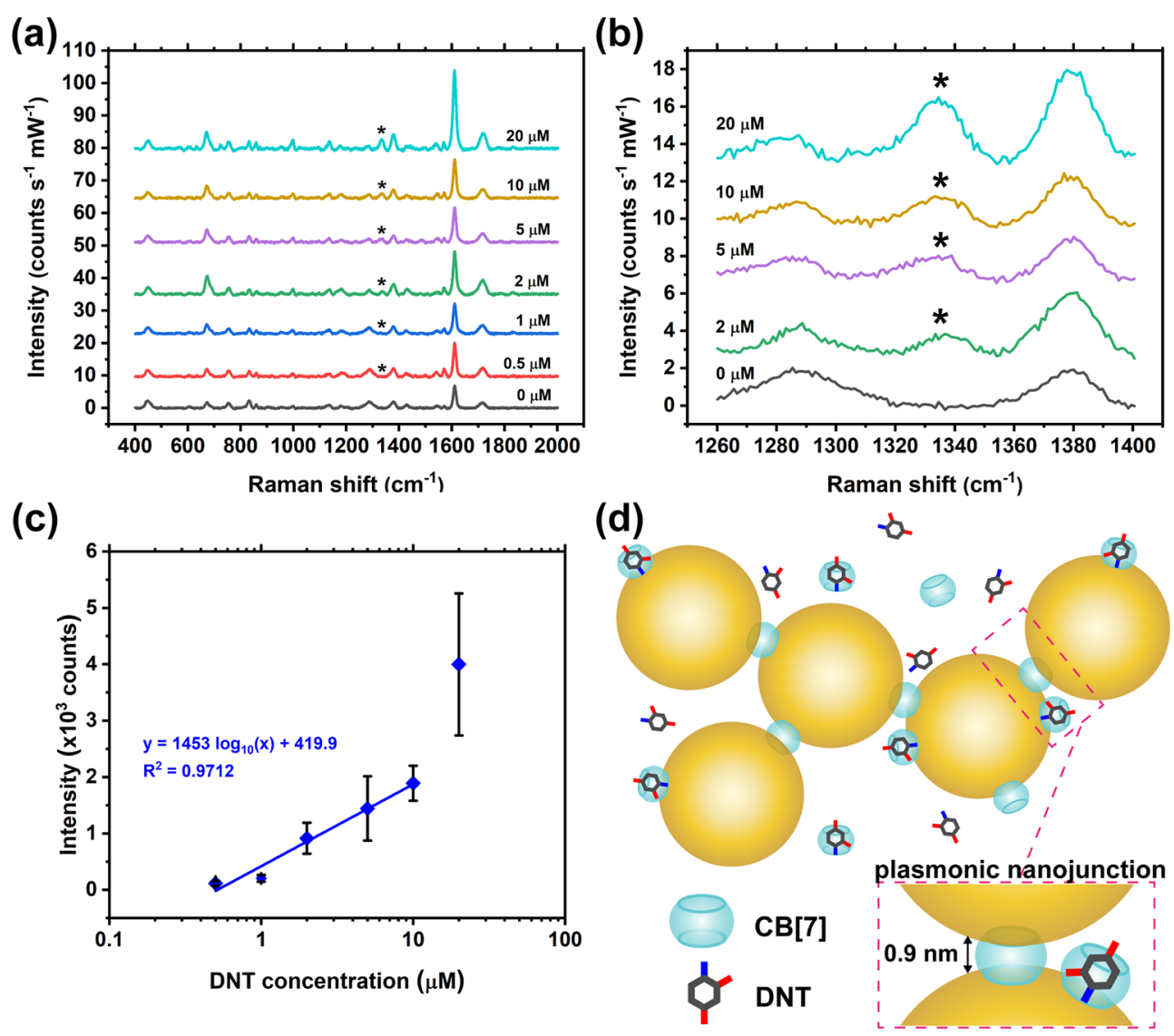

Figure 3. (a) Full-range and (b) zoom-in SERS spectra of DNT with concentrations from 0 to $20 \mu \mathrm{M}$; aggregation of Au NPs was triggered by 10 $\mu \mathrm{M}$ CB [7]. Spectra were baseline corrected and offset for clarity. (c) Corresponding plot of SERS intensity of the DNT main peak (marked by * in (a)) against DNT concentration. Logarithmic fittings were performed to reveal correlation between SERS intensity and DNT concentration from 0.5 to $10 \mu \mathrm{M}$ DNT. (d) Schematic illustration of precise plasmonic nanojunctions within dynamic aggregates of Au NPs formed by CB[7] for DNT detection (not to scale).

wB97X-D/6-311+G**). All quantum mechanical calculations were done using restricted (closed-shell) models. The binding energy of the discrete $\mathrm{CB}[7]-\mathrm{DNT}$ inclusion complex was obtained by subtracting the energies of $\mathrm{CB}[7]$ and free DNT from that of the complex optimized and calculated at the same level of theory. Raman spectra were simulated at the HF/3-
$21 \mathrm{G}$ level of theory as previously reported. ${ }^{24}$ A scaling factor of 0.90 was applied to correct the calculated vibrational frequencies.

UV-Visible Binding Titration. UV-vis measurements were performed on a Varian Cary 4000 UV-visible spectrophotometer. DNT solution $(10 \mu \mathrm{M}$ in water, $\mathrm{pH} 7)$ 
(a)

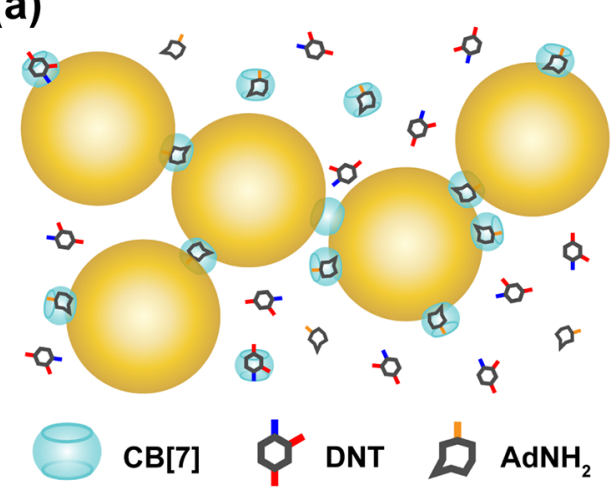

(c)

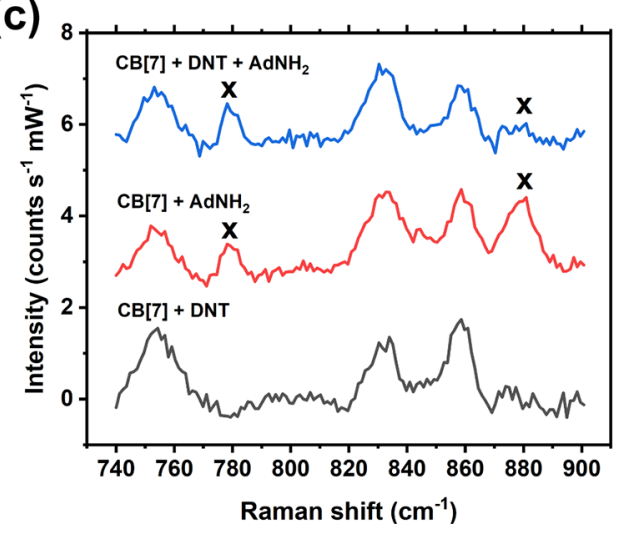

(b)

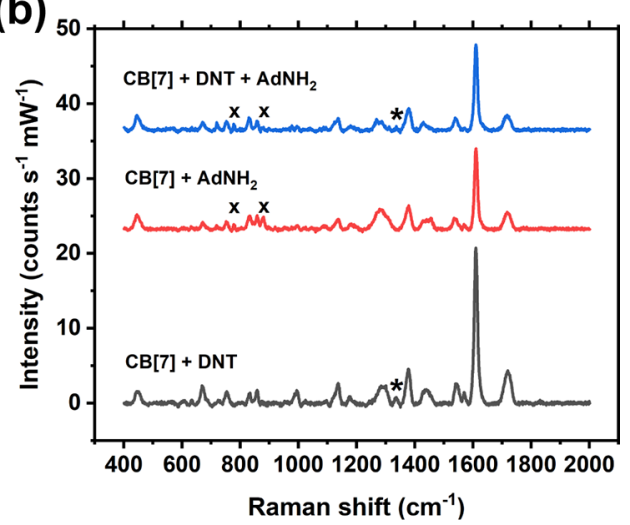

(d)

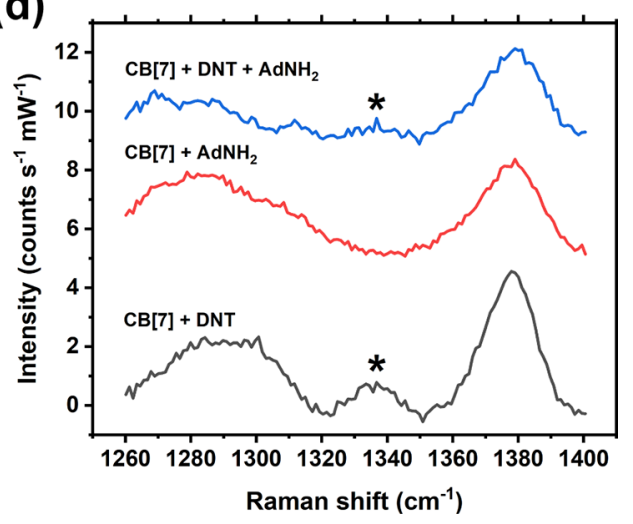

Figure 4. (a) Schematic illustration of competitive formation of host-guest complexes of $\mathrm{AdNH}_{2}$ and DNT with CB[7]. (b) Full-range and (c,d) zoom-in SERS spectra showing competitive formation of host-guest complexes of $\mathrm{AdNH}_{2}$ and DNT with CB[7]. (d) The DNT signal dropped to $\sim 33 \%$ when $10 \mu \mathrm{M} \mathrm{AdNH}{ }_{2}$ was added to $10 \mu \mathrm{M} \mathrm{CB}[7]-\mathrm{DNT}$. Main Raman peaks of DNT and $\mathrm{AdNH}_{2}$ are marked by $*$ and $\mathrm{x}$, respectively. Spectra were baseline corrected and offset for clarity.

was placed in a rectangular cuvette with $1 \mathrm{~cm}$ optical path length to which small amounts of the $\mathrm{CB}[7]$ stock solution (1 $\mathrm{mM}$ ) were added up to 4-5 equiv, such that the total concentration of DNT was kept constant, while that of the $\mathrm{CB}[7]$ was gradually increased.

Isothermal Titration Calorimetry (ITC). ITC experiments were carried out in water (unbuffered) on a VP-ITC from Microcal, Inc., at $25{ }^{\circ} \mathrm{C}$. The binding equilibria were studied using a cellular guest (DNT) concentration of $0.1 \mathrm{mM}$, to which a $1.0 \mathrm{mM} \mathrm{CB}[7]$ solution was titrated. Typically, 27 consecutive injections of $10 \mu \mathrm{L}$ were used. All solutions were degassed prior to titration. The first data point was removed from the data set prior to curve fitting according to a one-setof-sites model. The knowledge of the complex stability constant $\left(K_{\mathrm{a}}\right)$ and molar reaction enthalpy $\left(\Delta H^{\circ}\right)$ enabled the calculation of the standard free energy $\left(\Delta G^{\circ}\right)$ and entropy changes $\left(\Delta S^{\circ}\right)$ according to $\Delta G^{\circ}=-R T \ln K_{\mathrm{a}}=\Delta H^{\circ}-$ $T \Delta S^{\circ}$.

Raman and Surface-Enhanced Raman Spectroscopy (SERS). Raman and SERS spectra were acquired using a Reinshaw Raman InVia Microscope with a $633 \mathrm{~nm} \mathrm{He}-\mathrm{Ne}$ laser $(11.36 \mathrm{~mW})$ and focused onto the sample via a $50 \times$ objective lens (N.A. $=0.75)$. The grating used was 1800 lines $\mathrm{mm}^{-1}$, giving a spectral resolution of $1 \mathrm{~cm}^{-1}$. All spectra were calibrated with respect to $\mathrm{Si}$ and acquired at room temperature. Stock solutions were prepared by mixing $\mathrm{CB}[7]$ and DNT and, in the case of control experiments, in the presence of $\mathrm{AdNH}_{2}$, 4-MBA, or 4-MPBA. Similarly, CB[7]-PA stock solution was prepared by mixing $\mathrm{CB}[7]$ and PA. A $20 \mu \mathrm{L}$ drop of the premixed stock solution was added to a $180 \mu \mathrm{L} A u \mathrm{NP}$ solution in a $0.5 \mathrm{~mL}$ Eppendorf tube. The sample solution was stirred for $30 \mathrm{~s}$ before dropping $15 \mu \mathrm{L}$ onto a microscopic slide for SERS measurements. Three accumulations of $30 \mathrm{~s}$ scan were acquired on each measurement. Five measurements were taken across different regions of interest per sample. The spectra were averaged and baseline corrected using an asymmetric least-squares (ALS) plugin in Origin.

\section{RESULTS AND DISCUSSION}

Supramolecular Chemistry of $\mathrm{CB}[7]$ and DNT. The host-guest complexation of $\mathrm{CB}[7]$ and DNT was first verified by ${ }^{1} \mathrm{H}$ NMR in $\mathrm{D}_{2} \mathrm{O}$ which shows characteristic upfield shifts of the DNT proton signals (Figure S2). NMR titration experiments further revealed that the complexation-dissociation dynamics falls into the slow exchange regime on the NMR time scale at $298 \mathrm{~K}$ (Figure S3). The change in chemical shift $(\Delta \delta)$ after inclusion complex formation for $H_{\mathrm{d}} / H_{\mathrm{c}}\left(\Delta \delta_{\mathrm{d}}=\right.$ $-0.86 \mathrm{ppm}$ and $\left.\Delta \delta_{\mathrm{c}}=-1.1 \mathrm{ppm}\right)$ of DNT is larger than for $H_{\mathrm{b}} / H_{\mathrm{a}}\left(\Delta \delta_{\mathrm{b}}=-0.12 \mathrm{ppm}\right.$ and $\left.\Delta \delta_{\mathrm{a}}=-0.7 \mathrm{ppm}\right)$, which indicates that $H_{\mathrm{d}} / H_{\mathrm{c}}$ are located deep inside the $\mathrm{CB}[7]$ cavity, whereas $H_{\mathrm{b}} / H_{\mathrm{a}}$ are situated closer to the carbonyl rim. Interestingly, the anisotropic binding of DNT leads to desymmetrization of the $\mathrm{CB}[7]$ protons and therefore splits the corresponding NMR signals in the complex. The NMRdeduced binding geometry matches well with the energyminimized molecular model of the $\mathrm{CB}[7]-\mathrm{DNT}$ complex calculated at the CPCM/wB97XD/6-311+G** level of theory (Figure 1d). The binding energy of a discrete complex is 
(a)

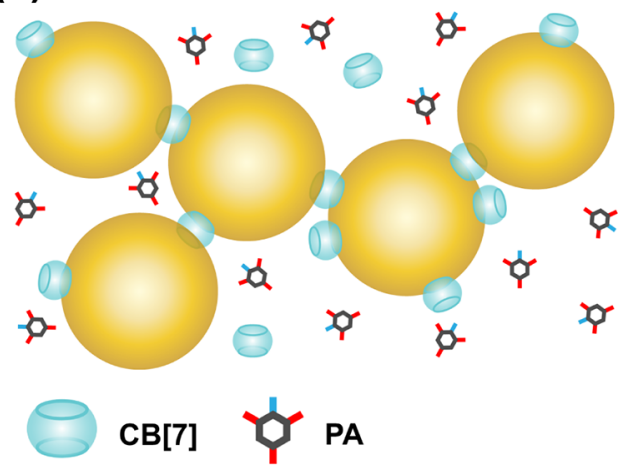

(c)

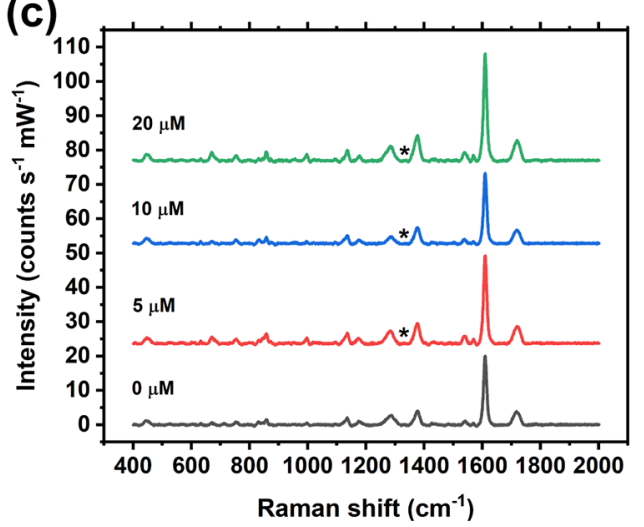

(b)

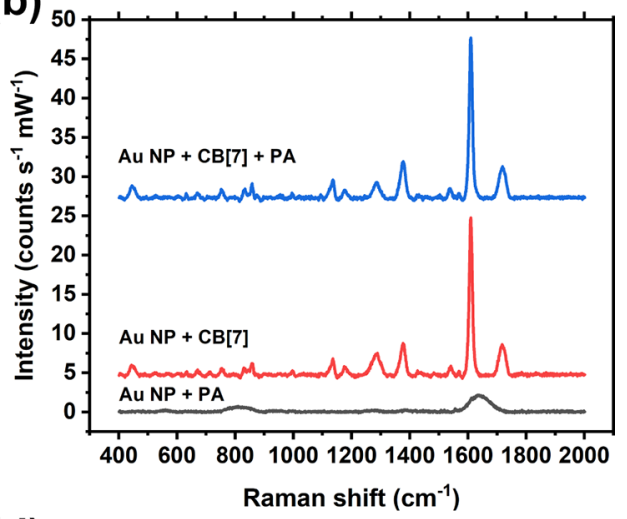

(d)

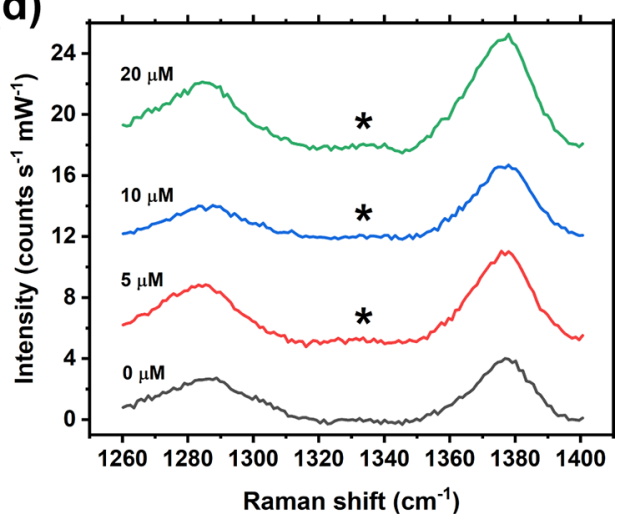

Figure 5. (a) Schematic illustration showing host-guest complexes of $\mathrm{CB}[7]$ and PA cannot be formed due to the large molecular size of PA. (b) SERS spectra of Au NPs and PA in the presence and absence of CB[7]. (c) Full-range and (d) zoom-in SERS spectra of PA with concentrations from $0 \mu \mathrm{M}$ to $20 \mu \mathrm{M}$ in the presence of $10 \mu \mathrm{M} \mathrm{CB}[7]$. Spectra were baseline corrected and offset for clarity.

calculated to be $-26.9 \mathrm{kcal} \mathrm{mol}^{-1}$ (see SI for details), which is consistent to that of other similar neutral complexes. ${ }^{30}$ It is noted that the $\mathrm{NO}_{2}$ group at the 4-position is sticking out through the portal, blocking its potential interaction with $\mathrm{Au}$ NPs. Nevertheless, the other CB portal remains accessible, leaving it available to bind to the surface of Au NPs which is critical to the subsequent SERS study.

It was challenging to extract an accurate binding constant with $\mathrm{CB}[7]$ using the NMR titration method, owing to the relatively low solubility of DNT in water $(0.19 \mathrm{mg} / \mathrm{mL}$ at 22 $\left.{ }^{\circ} \mathrm{C}\right)^{31}$ and the relatively high aqueous binding constant (see below). Hence, the system was further characterized by UVvis titration, and a binding constant for $\mathrm{CB}[7]$ and $\mathrm{DNT}$ of $(1.6 \pm 0.2) \times 10^{6} \mathrm{M}^{-1}$ was obtained (Figure $2 \mathrm{a}$ ). We also determined the thermodynamics of binding by using isothermal titration calorimetry (ITC) measurements for DNT binding with $\mathrm{CB}[7]$ and verified the postulated 1:1 binding mode. The binding constant was found to be $(4.94 \pm$ $0.34) \times 10^{5} \mathrm{M}^{-1}$; within methodological error, this affinity is consistent with the UV-vis titration results. The ITC data also revealed that the binding is enthalphically driven $\left(\Delta H^{\circ}=\right.$ $\left.-11.37 \mathrm{kcal} \mathrm{mol}^{-1}\right)$. The results indicate that displacement of high-energy water molecules by DNT is the major driving force for complex formation, which is typical for $\mathrm{CB}$ [7] hostguest complexes (Figure $2 \mathrm{~b}$ ). ${ }^{32}$ Additionally, dispersion interactions between the highly polarizable nitroaromatics and the hydrophobic cavity are likely contributors. ${ }^{33,34}$ Negative entropic contributions $\left(\Delta S^{\circ}=-0.012 \mathrm{kcal} \mathrm{mol}^{-1}\right.$ $\mathrm{K}^{-1}$ or $T \Delta S^{\circ}=-3.61 \mathrm{kcal} \mathrm{mol}^{-1}$ ) reflect the formation of a conformationally restricted, tight inclusion complex, which is consistent with the deformed, ellipsoidal shape of the $\mathrm{CB}[7]$ moiety within the complex in the energy-minimized molecular model (Figure 1d).

The Raman spectrum of solid DNT is characterized by a strong peak at $1348 \mathrm{~cm}^{-1}$ corresponding to symmetric $\mathrm{NO}_{2}$ stretching. ${ }^{35}$ The two peaks at 1544 and $1611 \mathrm{~cm}^{-1}$ correspond to asymmetric $\mathrm{NO}_{2}$ stretching and aromatic $\mathrm{NO}_{2}$ conjugation, respectively. ${ }^{35}$ The Raman spectrum of solid $\mathrm{CB}[7]$ is characterized by two major peaks at 444 and 833 $\mathrm{cm}^{-1}$ which are assigned to the ring scissor and ring deformation modes, respectively. ${ }^{24}$ The Raman spectrum of the 1:1 CB[7]-DNT host-guest complex in aqueous solution shows slight shifts in peak positions compared to the Raman spectra of $\mathrm{CB}[7]$ and DNT powder (Figure S4a). DNT signals are dominant in the Raman spectrum of the $\mathrm{CB}[7]-\mathrm{DNT}$ complex, as DNT is more Raman-active than $\mathrm{CB}[7]$. Raman spectra of $\mathrm{CB}[7], \mathrm{DNT}$, and their complex in the gas phase were modeled at the HF/3-21G level of theory (Figure S4b), showing general consistency with the experimental data. The Raman spectrum of the complex is roughly equal to the sum of that of CB[7] and DNT with slight shifts in some of the peaks.

SERS Sensing of the CB[7]-DNT Host-Guest Complex. The potential detection of the trace-explosive marker DNT by SERS was investigated by adding a solution of preformed 1:1 CB[7]-DNT complex to a solution of citratestabilized Au NPs of $40 \mathrm{~nm}$ size (Figure 1b). CB-mediated aggregation of Au NPs was observed, evidenced by the change in solution color from red to purple and the transmission electron microscopy (TEM) images; this is consistent with our previous findings. ${ }^{20-22}$ A SERS titration was first performed by 

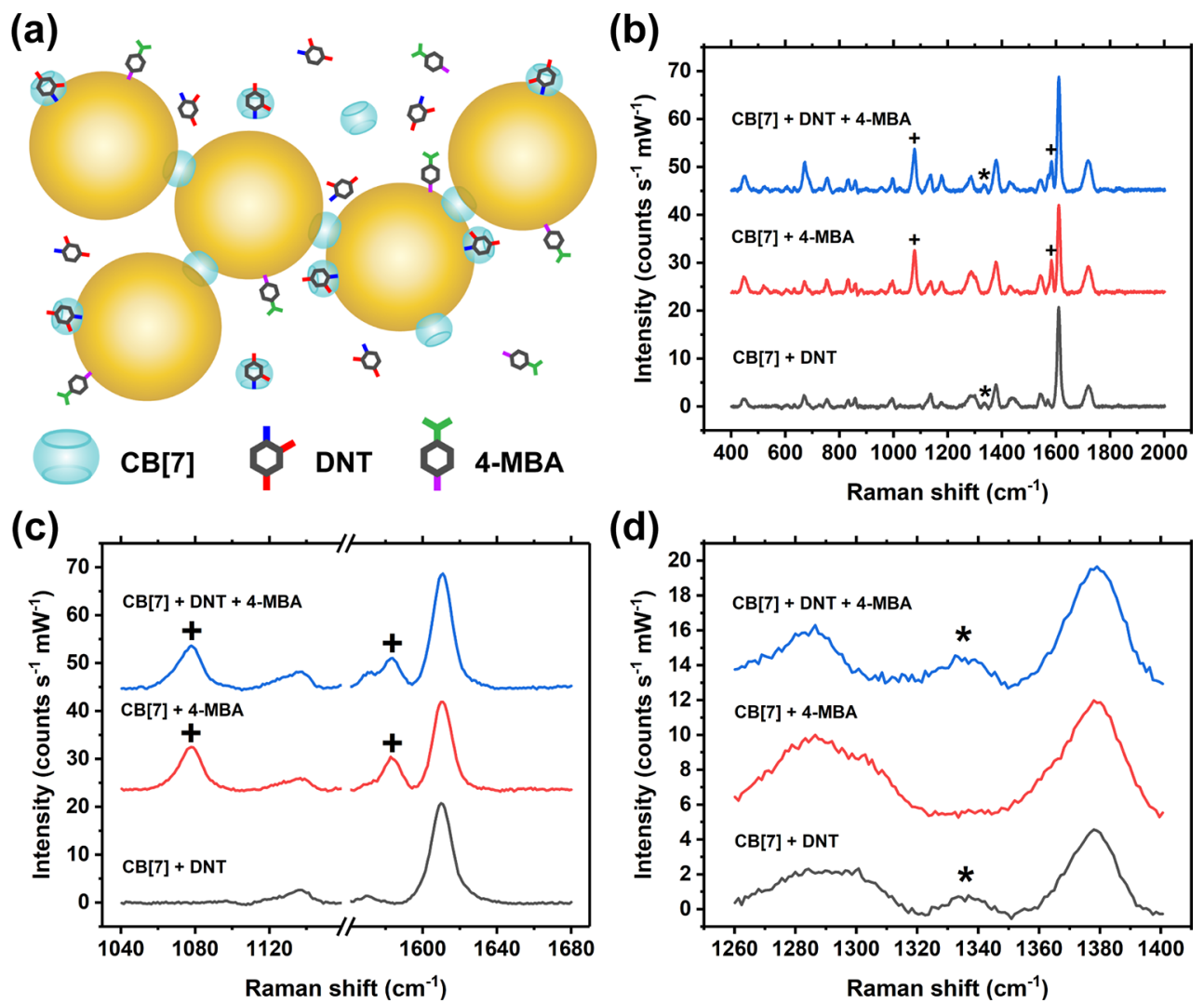

Figure 6. (a) Schematic illustration of the precise nanojunctions between adjacent Au NPs formed by CB[7] with DNT being encapsulated inside their hydrophobic cavities and 4-MBA being strongly bound to Au NPs (not to scale). (b) Full-range and (c,d) zoom-in SERS spectra of a $10 \mu \mathrm{M}$ $\mathrm{CB}[7]-\mathrm{DNT}$ 1:1 inclusion complex in the presence of a strong ligand, $1 \mu \mathrm{M}$ 4-mercaptobenezonic acid (4-MBA). Main Raman peaks of DNT and 4-MBA are marked by $*$ and + , respectively. Spectra were baseline corrected and offset for clarity.

adding different concentrations of $\mathrm{CB}[7]-\mathrm{DNT}$ complex (Figure S5a,b). The characteristic Raman peak of DNT at $1334 \mathrm{~cm}^{-1}$ remained readily observable for DNT concentrations down to $\sim 10 \mu \mathrm{M}$. Nevertheless, at very low concentration of the complex, no aggregation of $\mathrm{Au}$ NPs, and therefore no SERS effect, can be observed because of the insufficient amount of $\mathrm{CB}[7]$ to trigger the self-assembly process. It is noted that the broad peak at $\sim 1640 \mathrm{~cm}^{-1}$ in the Raman spectrum of DNT is due to the $\mathrm{H}-\mathrm{O}-\mathrm{H}$ bending mode of liquid water (Figure S6). ${ }^{36}$

To test the detection limit, we performed SERS titrations of $\mathrm{DNT}$ at a constant $\mathrm{CB}[7]$ concentration of $10 \mu \mathrm{M}$ (Figure $3 a, b)$. This condition ensures the formation of reproducible aggregates, i.e., SERS substrates, because the aggregation kinetics is determined by the $\mathrm{CB}: \mathrm{Au} \mathrm{NP}$ ratio. ${ }^{22}$ The DNT main peak related to the symmetric $\mathrm{NO}_{2}$ stretching band can be observed clearly in the SERS spectra even when the concentration of DNT is as low as $2 \mu \mathrm{M}$ (Figure $3 \mathrm{~b}$ ). The detection limit of DNT was found to be $\sim 1 \mu \mathrm{M}$ with high linearity of the diagnostic Raman intensity $\left(R^{2} \sim 0.97\right)$ across a wide concentration range (Figure $3 \mathrm{c}$ ), which is comparable to other solution-based SERS techniques. ${ }^{3,28}$

$\mathrm{CB}[7]$ plays two critical roles in enhancing the SERS signals of DNT. First, its rigid molecular structure defines precise nanojunctions between the Au NPs, resulting in strong and localized plasmonic hotspots. This effect is supported by a control experiment using $0.1 \mathrm{M} \mathrm{NaCl}$ as the aggregating agent (Figure S7). In this case, the Raman features are significantly broader, and no correlation between Raman intensity and
DNT concentration can be observed, owing to the random Au NP aggregates from uncontrolled aggregation. Second, surfacebound $\mathrm{CB}[7]$ can encapsulate DNT molecules, leading to an enriched DNT concentration on the Au NP surface and around the plasmonic hotspots. This effect is especially important for analytes, such as nitroaromatics, that have a low intrinsic affinity to gold surfaces, as opposed to many of the precedent examples using analytes with high affinity to gold. $^{27,28}$ The surface-enrichment effect was verified by a competitive binding experiment using 1-adamantylamine $\left(\mathrm{AdNH}_{2}\right)$ which is a strong binder to $\mathrm{CB}[7]$ (Figure 4). The DNT signal was reduced by $67 \%$ in the presence of equimolar $\mathrm{AdNH}_{2}$ which effectively displaces DNT from the $\mathrm{CB}[7]$ cavity.

Selectivity and Robustness of the Nitroaromatic Explosive SERS Sensor. The selectivity of our SERS system was demonstrated by control experiments with a structurally similar nitroaromatic explosive, picric acid (PA), which is too bulky to fit inside the $\mathrm{CB}[7]$ cavity as confirmed by NMR measurements (Figure S8). Its negative overall charge and high solubility in water also disfavor the binding with $\mathrm{CB}[7]$. Despite being 3.5 times more Raman active than DNT (Figure S9), PA does not exhibit any observable SERS signals in the presence of $\mathrm{CB}[7]$ (Figure 5), illustrating the importance of host-guest affinity in our sensing scheme.

Notably, in the CB[7]-DNT-Au NP system, aggregation of Au NPs should be mostly caused by empty $\mathrm{CB}[7]$, which is present in the system at dynamic equilibrium of host-guest complexation. In contrast to a number of previous reports 
using small analyte molecules that can be entirely encapsulated deep inside the $\mathrm{CB}$ cavity, the $\mathrm{CB}[7]-\mathrm{DNT}$ complex alone could not mediate the aggregation of Au NPs because one of the $\mathrm{CB}[7]$ portals is blocked by the bulky nitro group, as evidenced by the NMR data and the energy-minimized molecular model of the complex (Figure 1d). Nevertheless, $\mathrm{CB}[7]-\mathrm{DNT}$ complexes can still stay close to the plasmonic hot-spots through binding to the Au NP surface via the accessible portal (Figure 3d). This phenomenon is reflected in the relatively large error bar and the slight departure from linearity in the sample with excess DNT $(20 \mu \mathrm{M}$ DNT and 10 $\mu \mathrm{M} \mathrm{CB}[7])$ in Figure $3 \mathrm{c}$, where the concentration of empty $\mathrm{CB}[7]$ is lower than that in other samples due to a shift in equilibrium position by excess DNT.

Finally, as a proof-of-concept experiment, we performed the SERS analysis of DNT in the presence of strong Au NP ligands, 4-mercaptobenezoic acid (4-MBA) and 4-mercaptophenylboronic acid (4-MPBA), to mimic strongly binding environmental impurities that might be featured in field applications. Although 4-MBA can bind strongly to Au NPs, no SERS signal of $1 \mu \mathrm{M} 4-\mathrm{MBA}$ can be observed in the absence of $\mathrm{CB}[7]$ (Figure $\mathrm{S} 10 \mathrm{a}, \mathrm{c}, \mathrm{d}$ ). $\mathrm{CB}[7]$ forms nanojunctions between $\mathrm{Au}$ NPs as aforementioned and gives rise to the SERS signals of 4-MBA (Figure 6a). We investigated multiplexed SERS detection of $10 \mu \mathrm{M} \mathrm{DNT}$ and $1 \mu \mathrm{M} 4-\mathrm{MBA}$ in the presence of $\mathrm{CB}[7]$. The characteristic peaks of $4-\mathrm{MBA}$ and DNT are clearly observed in the SERS spectra (Figure $6 \mathrm{~b}-\mathrm{d}$ ). Similar results were obtained in the case of 4-MPBA, which reflects the tolerance of our SERS system in detecting trace explosives (Figure S10b,e,f). By extracting Raman shift information, interference from various contaminants can be eliminated, in contrast to classical on/off-type sensing techniques which are more prone to false-positive results.

\section{CONCLUSIONS}

In this work, we report a novel sensor that exploits the formation of a neutral host-guest complex between $\mathrm{CB}[7]$ and the explosive marker DNT. Key binding parameters of the complexation have been quantified using NMR, UV-vis titrations, and ITC measurements, supported by molecular modeling based on DFT. While CB[7] is known to host a wide range of positively charged guest motifs, neutral guests are much rarer. Our findings demonstrate new possibilities in utilizing aqueous host-guest chemistry of $\mathrm{CB}$ [7] for polysubstituted nitroaromatics, which is an important class of explosive compounds. The complexation between $\mathrm{CB}[7]$ and DNT allows a sensing scheme based on aqueous SERS, offering rapid response, high reproducibility, and signal linearity. The system achieved an explosive detection limit of $\sim 1 \mu \mathrm{M}$, consistent with other solution-based SERS sensors. Notably, the molecular recognition and host-guest binding properties of $\mathrm{CB}[7]$ are retained after anchoring onto the surface of Au NPs, which has been verified by a competitive binding experiment and a negative control experiment using picric acid. Proof-of-concept experiments showed that the SERS sensor is tolerant against the presence of model organic contaminants, paving the way toward its potential application in on-site environmental monitoring.

\section{ASSOCIATED CONTENT}

\section{S Supporting Information}

The Supporting Information is available free of charge on the ACS Publications website at DOI: 10.1021/acs.jpcc.9b02363.
Computational details of $\mathrm{CB}[7]-\mathrm{DNT}$; TEM images of $\mathrm{Au} \mathrm{NP}-\mathrm{CB}[7]-\mathrm{DNT}$; NMR spectra of $\mathrm{CB}[7]-\mathrm{DNT}$ and $\mathrm{CB}[7]-\mathrm{PA}$; Raman spectra of $\mathrm{DNT}, \mathrm{CB}[7]$, $\mathrm{CB}[7]-\mathrm{DNT}$, water, $\mathrm{Au} \mathrm{NPs}, \mathrm{AdNH}_{2}, \mathrm{PA}, 4-\mathrm{MBA}$, and 4-MPBA; and SERS spectra of $\mathrm{CB}[7]-\mathrm{DNT}, \mathrm{NaCl}$ control, 4-MBA, and 4-MPBA (PDF)

\section{AUTHOR INFORMATION}

\section{Corresponding Author}

*E-mail: tungchun.lee@ucl.ac.uk.

\section{ORCID}

William J. Peveler: 0000-0002-9829-2683

Khaleel I. Assaf: 0000-0003-4331-8492

Werner M. Nau: 0000-0002-7654-6232

Tung-Chun Lee: 0000-0002-3163-0000

\section{Author Contributions}

WIKC, WJP, and TCL designed the experiments. WIKC performed all Raman and SERS measurements. SM and WJP performed NMR measurements. WIKC, SM, and TCL conducted the TEM imaging. Binding studies using UV-vis titration and ITC were performed by KIA. Computational studies were done by TCL. All authors contributed to data analysis and to writing of the manuscript.

\section{Notes}

The authors declare no competing financial interest.

\section{ACKNOWLEDGMENTS}

SM and TCL are grateful to the Research Project Grant (RPG2016-393) funded by the Leverhulme Trust. WIKC, IPP, MO, and TCL are grateful to the Studentship funded by the A*STAR-UCL Research Attachment Programme through the EPSRC M3S CDT (EP/L015862/1). WJP acknowledges the University of Glasgow for a Lord Kelvin Adam Smith Fellowship. KIA and WMN thank the DFG for grant NA$686 / 8$ within the priority program SPP 1807 "Control of London Dispersion Interactions in Molecular Chemistry". WIKC and MO acknowledge the SBIC LBOI lab for use of facilities and resources.

\section{REFERENCES}

(1) Peveler, W. J.; Roldan, A.; Hollingsworth, N.; Porter, M. J.; Parkin, I. P. Multichannel Detection and Differentiation of Explosives with a Quantum Dot Array. ACS Nano 2016, 10, 1139-1146.

(2) Peveler, W. J.; Jaber, S. B.; Parkin, I. P. Nanoparticles in Explosives Detection - the State-of-the-Art and Future Directions. Forensic Sci., Med., Pathol. 2017, 13, 490-494.

(3) Cecchini, M. P.; Turek, V. A.; Paget, J.; Kornyshev, A. A.; Edel, J. B. Self-Assembled Nanoparticle Arrays for Multiphase Trace Analyte Detection. Nat. Mater. 2013, 12, 165-171.

(4) Thomas, S. W.; Joly, G. D.; Swager, T. M. Chemical Sensors Based on Amplifying Fluorescent Conjugated Polymers. Chem. Rev. 2007, 107, 1339-1386.

(5) Zapata, F.; López-López, M.; García-Ruiz, C. Detection and Identification of Explosives by Surface Enhanced Raman Scattering. Appl. Spectrosc. Rev. 2016, 51, 227-262.

(6) Zhu, W.; Li, W.; Wang, C.; Cui, J.; Yang, H.; Jiang, Y.; Li, G. $\mathrm{CB}[8]$-Based Rotaxane as a Useful Platform for Sensitive Detection and Discrimination of Explosives. Chem. Sci. 2013, 4, 3583-3590.

(7) Zhu, W.; Wang, C.; Li, W.; Tao, C.-a.; Cui, J.; Yang, H.; Jiang, Y.; Li, G. A New Strategy for Selective Detection of Nitrated Explosives Based on a Confinement Effect of Nanocavity. J. Mater. Chem. A 2013, 1, 11741-11747. 
(8) Liu, X.; Zhao, L.; Shen, H.; Xu, H.; Lu, L. Ordered Gold Nanoparticle Arrays as Surface-Enhanced Raman Spectroscopy Substrates for Label-Free Detection of Nitroexplosives. Talanta 2011, 83, 1023-1029.

(9) Smith, K. D.; McCord, B. R.; MacCrehan, W. A.; Mount, K.; Rowe, W. F. Detection of Smokeless Powder Residue on Pipe Bombs by Micellar Electrokinetic Capillary Electrophoresis. J. Forensic Sci. 1999, 44, 789-794.

(10) Amaral, H. I. F.; Fernandes, J.; Berg, M.; Schwarzenbach, R. P.; Kipfer, R. Assessing TNT and DNT Groundwater Contamination by Compound-Specific Isotope Analysis and ${ }^{3} \mathrm{H}-{ }^{3} \mathrm{He}$ Groundwater Dating: A Case Study in Portugal. Chemosphere 2009, 77, 805-812.

(11) Berg, M.; Bolotin, J.; Hofstetter, T. B. Compound-Specific Nitrogen and Carbon Isotope Analysis of Nitroaromatic Compounds in Aqueous Samples Using Solid-Phase Microextraction Coupled to GC/IRMS. Anal. Chem. 2007, 79, 2386-2393.

(12) Kalderis, D.; Juhasz, A. L.; Boopathy, R.; Comfort, S. Soils Contaminated with Explosives: Environmental Fate and Evaluation of State-of-the-Art Remediation Processes (IUPAC Technical Report). Pure Appl. Chem. 2011, 83, 1407-1484.

(13) Chen, W.; Zuckerman, N. B.; Konopelski, J. P.; Chen, S. Pyrene-Functionalized Ruthenium Nanoparticles as Effective Chemosensors for Nitroaromatic Derivatives. Anal. Chem. 2010, 82, 461465.

(14) Jiang, Y.; Zhao, H.; Zhu, N.; Lin, Y.; Yu, P.; Mao, L. A Simple Assay for Direct Colorimetric Visualization of Trinitrotoluene at Picomolar Levels Using Gold Nanoparticles. Angew. Chem., Int. Ed. 2008, 47, 8601-8604.

(15) Riskin, M.; Tel-Vered, R.; Bourenko, T.; Granot, E.; Willner, I. Imprinting of Molecular Recognition Sites through Electropolymerization of Functionalized Au Nanoparticles: Development of an Electrochemical TNT Sensor Based on Pi-Donor-Acceptor Interactions. J. Am. Chem. Soc. 2008, 130, 9726-9733.

(16) Matsumoto, K.; Torimaru, A.; Ishitobi, S.; Sakai, T.; Ishikawa, H.; Toko, K.; Miura, N.; Imato, T. Preparation and Characterization of a Polyclonal Antibody from Rabbit for Detection of Trinitrotoluene by a Surface Plasmon Resonance Biosensor. Talanta 2005, 68, 305311.

(17) Fang, Y.; Seong, N.-H.; Dlott, D. D. Measurement of the Distribution of Site Enhancements in Surface-Enhanced Raman Scattering. Science 2008, 321, 388-392.

(18) Ben-Jaber, S.; Peveler, W. J.; Quesada-Cabrera, R.; Cortés, E.; Sotelo-Vazquez, C.; Abdul-Karim, N.; Maier, S. A.; Parkin, I. P. Photo-Induced Enhanced Raman Spectroscopy for Universal UltraTrace Detection of Explosives, Pollutants and Biomolecules. Nat. Commun. 2016, 7, 12189.

(19) Ben-Jaber, S.; Peveler, W. J.; Quesada-Cabrera, R.; Sol, C. W. O.; Papakonstantinou, I.; Parkin, I. P. Sensitive and Specific Detection of Explosives in Solution and Vapour by Surface-Enhanced Raman Spectroscopy on Silver Nanocubes. Nanoscale 2017, 9, 16459-16466.

(20) Lee, T.-C.; Scherman, O. A. Formation of Dynamic Aggregates in Water by Cucurbit[5] uril Capped with Gold Nanoparticles. Chem. Commun. 2010, 46, 2438-2440.

(21) Lee, T.-C.; Scherman, O. A. A Facile Synthesis of Dynamic Supramolecular Aggregates of Cucurbit $[n]$ uril $(\mathrm{n}=5-8)$ Capped with Gold Nanoparticles in Aqueous Media. Chem. - Eur. J. 2012, 18, $1628-1633$.

(22) Taylor, R. W.; Lee, T.-C.; Scherman, O. A.; Esteban, R.; Aizpurua, J.; Huang, F. M.; Baumberg, J. J.; Mahajan, S. Precise Subnanometer Plasmonic Junctions for SERS within Gold Nanoparticle Assemblies Using Cucurbit[n]uril "Glue". ACS Nano 2011, 5, 3878-3887.

(23) Nau, W. M.; Florea, M.; Assaf, K. I. Deep Inside Cucurbiturils: Physical Properties and Volumes of their Inner Cavity Determine the Hydrophobic Driving Force for Host-Guest Complexation. Isr. J. Chem. 2011, 51, 559-577.

(24) Mahajan, S.; Lee, T.-C.; Biedermann, F.; Hugall, J. T.; Baumberg, J. J.; Scherman, O. A. Raman and SERS Spectroscopy of Cucurbit $[n]$ urils. Phys. Chem. Chem. Phys. 2010, 12, 10429-10433.
(25) Tao, C.-a.; An, Q.; Zhu, W.; Yang, H.; Li, W.; Lin, C.; Xu, D.; Li, G. Cucurbit $[n]$ urils as a SERS Hot-Spot Nanocontainer through Bridging Gold Nanoparticles. Chem. Commun. 2011, 47, 9867-9869.

(26) Kasera, S.; Biedermann, F.; Baumberg, J. J.; Scherman, O. A.; Mahajan, S. Quantitative SERS Using the Sequestration of Small Molecules Inside Precise Plasmonic Nanoconstructs. Nano Lett. 2012, 12, 5924-5928.

(27) Taylor, R. W.; Coulston, R. J.; Biedermann, F.; Mahajan, S.; Baumberg, J. J.; Scherman, O. A. In Situ SERS Monitoring of Photochemistry within a Nanojunction Reactor. Nano Lett. 2013, 13, 5985-5990.

(28) Kasera, S.; Herrmann, L. O.; Barrio, J. d.; Baumberg, J. J.; Scherman, O. A. Quantitative Multiplexing with Nano-SelfAssemblies in SERS. Sci. Rep. 2015, 4, 6785.

(29) Lagona, J.; Mukhopadhyay, P.; Chakrabarti, S.; Isaacs, L. The Cucurbit[n] uril Family. Angew. Chem., Int. Ed. 2005, 44, 4844-4870.

(30) Lee, T.-C.; Kalenius, E.; Lazar, A. I.; Assaf, K. I.; Kuhnert, N.; Grün, C. H.; Jänis, J.; Scherman, O. A.; Nau, W. M. Chemistry inside Molecular Containers in the Gas Phase. Nat. Chem. 2013, 5, 376382.

(31) Phelan, J. M.; Barnett, J. L. Solubility of 2,4-Dinitrotoluene and 2,4,6-Trinitrotoluene in Water. J. Chem. Eng. Data 2001, 46, 375376.

(32) Biedermann, F.; Uzunova, V. D.; Scherman, O. A.; Nau, W. M.; De Simone, A. Release of High-Energy Water as an Essential Driving Force for the High-Affinity Binding of Cucurbit $[n]$ urils. J. Am. Chem. Soc. 2012, 134, 15318-15323.

(33) Biedermann, F.; Nau, W. M.; Schneider, H.-J. The Hydrophobic Effect Revisited-Studies with Supramolecular Complexes Imply High-Energy Water as a Noncovalent Driving Force. Angew. Chem., Int. Ed. 2014, 53, 11158-11571.

(34) Liu, T.; Schneider, H.-J. Additivity and Quantification of Dispersive Interactions-from Cyclopropyl to Nitro Groups: Measurements on Porphyrin Derivatives. Angew. Chem., Int. Ed. 2002, 41, $1368-1370$.

(35) Fierro-Mercado, P. M.; Hernández-Rivera, S. P. Highly Sensitive Filter Paper Substrate for SERS Trace Explosives Detection. Int. J. Spectrosc. 2012, 2012, 7.

(36) Carey, D. M.; Korenowski, G. M. Measurement of the Raman Spectrum of Liquid Water. J. Chem. Phys. 1998, 108, 2669-2675. 